Jpn. J. Genet. (1994) 69, pp. 371-376

\title{
Identification of chromosomes involved in translocations in wild Emmer
}

\author{
Kozo Nishikawa ${ }^{1, *}$, Shima Mizuno ${ }^{2}$ and Yoshihiko Furuta ${ }^{2}$ \\ ${ }^{1}$ Aichi Sangyo University, 12-5 Harayama, Okamati, Okazaki 444 \\ ${ }^{2}$ Faculty of Agriculture, Gifu University, 1-1 Yanagido, Gifu 501-11, Japan
}

(Received 19 November 1993)

\begin{abstract}
With the aid of telocentric lines of Emmer wheat, the chromosomes involved in seven chromosome types (one standard type and six translocation types) in wild Emmer, Triticum dicoccoides, were identified. Type E1a was of almost the same chromosome structure as that of durum LD 222 with a small reciprocal translocation between chromosomes $4 \mathrm{~B}$ and $2 \mathrm{~B}$. Type E1b had a major translocation between $2 \mathrm{~A}$ and $2 \mathrm{~B}$ and a minor one probably between $2 \mathrm{~B}$ and $3 \mathrm{~B}$. Type E2 had a major translocation between $2 \mathrm{~B}$ and 3B. Type E3 had a major translocation between $5 \mathrm{~B}$ and $7 \mathrm{~B}$. Type $\mathrm{E} 4$ had a major translocation between $4 \mathrm{~B}$ and $3 \mathrm{~B}$ and a minor one between $2 \mathrm{~B}$ and $4 \mathrm{~B}$ or $3 \mathrm{~B}$. Type E5 had a major translocation between $6 \mathrm{~B}$ and $7 \mathrm{~B}$. Type $\mathrm{E} 6$ had a major translocation between $1 \mathrm{~A}$ and $5 \mathrm{~A}$. We discussed the result in comparison with the previously reported data on the same translocations.
\end{abstract}

\section{INTRODUCTION}

Five major and one minor types of translocation in wild Emmer, T. dicoccoides Körn. were reported by Kawahara and Tanaka (1983) and Kawahara (1984, 1986, 1987), who carried out the extensive studies on intraspecific variation, fundamental chromosome structure, and geographical distribution of the translocation types. Kawahara (1984), based on the chromosome pairing in the $\mathrm{F}_{1}$ 's between translocation testers and Einkorn wheat, estimated the genomes to which the chromosomes involved in the translocations belonged, but did not identify the translocation chromosomes themselves. As T. dicoccoides is a good source of the genes for agronomically important traits such as disease resistance, identification of chromosomes involved in the different types of translocation is desired from a viewpoint of cytogenetics and wheat breeding. Using telocentric lines of durum wheat, Nishikawa et al. (1986) identified the chromosomes involved in translocations among cultivated forms of Emmer wheat. The method was applied in this study to investigate the translocations found by Kawahara (1984).

* Corresponding author. 


\section{MATERIALS AND METHODS}

Seven tester strains of Triticum turgidum (L.) Thell. ssp. dicoccoides Körn., each representing Kawahara's seven chromosome structures (KU-1978B for Type E1a, KU-108-3 for E1b, KU-109 for E2, KU-195 for E3, KU-8915A for E4, KU-1945 for E5, KU-1952 for E6), were kindly provided by Dr. T. Kawahara, Plant Germ-plasm Institute, Faculty of Agriculture, Kyoto University.

Double ditelosomics $\left(13^{\prime \prime}+t^{\prime \prime}+t^{\prime \prime}\right)$ or ditelo-monotelosomics $\left(13^{\prime \prime}+t^{\prime \prime}+t^{\prime}\right)$ for the A and B genome chromosomes of a durum wheat [Triticum turgidum (L.) Thell. spp. turgidum conv. durum (Desf.) MK] cv. LD 222, which had been developed by the senior author, were crossed to the tester strains, and the chromosome pairing in PMC's of the $\mathrm{F}_{1}$ 's was observed.

\section{RESULTS AND DISCUSSION}

The telocentric chromosomes tended to fall apart from their homologous chromosome arms at metaphase I. This resulted in variations in the meiotic pairing of the telosomes. For example, a meiotic pairing $(\mathrm{t}+\mathrm{t}) 1^{\prime \prime \prime}$ (trivalent consisting of one whole and two telocentric chromosomes) dissociated into $t 1^{\prime \prime}+t^{\prime}$ and $2 t^{\prime}+1^{\prime}$, and the critical pairing $(t+t) 3^{V}$, which indicated the involvement of the telocentric chromosomes in the translocation, dissociated into $\mathrm{t} 3^{\mathrm{IV}}+\mathrm{t}^{\prime}, \mathrm{t} 2^{\prime \prime \prime}+$ $t 1^{\prime \prime}, 1^{\prime \prime \prime}+2 t^{\prime}$ and so on. In the monotelodisomic $F_{1}$ 's, the critical pairing, $\mathrm{t}^{\mathrm{IV}}$ occasionally dissociated into $t 1^{\prime \prime}+1^{\prime \prime}$, which can not be distinguished from normal pairing in non-critical $\mathrm{F}_{1}$ 's. So, this pairing type was recognized as critical in the lines which showed $\mathrm{t} 3^{\mathrm{IV}}$ in the other PMC's. The normal chromosomes were rarely formed two univalents or $1^{\prime \prime \prime}+1^{\prime}$. For the sake of brevity, the desynaptic pairing of the telosomes were scored as the complete pairings in Table 1: for example, $\mathrm{t} 1^{\prime \prime}+\mathrm{t}^{\prime}$ and $2 \mathrm{t}^{\prime}+\mathrm{t}^{\prime}$ were scored as $(\mathrm{t}+\mathrm{t}) 1^{\prime \prime \prime}$. Among telocentric chromosomes of LD222, the $1 \mathrm{~B}$ and $6 \mathrm{~B}$ telosomes showed a considerable degree of desynapsis in the $\mathrm{F}_{1}$ 's of all the testers. In the non-critical $\mathrm{F}_{1}$ 's, the quadrivalents occurred in a ring-of-four or chain-of-four configuration. The percentage of the ring-of-four was more than $60 \%$ in the $\mathrm{F}_{1}$ 's of the E2, E4, E5 and E6 testers, but it was about $20 \%$ in those of the E1a tester.

(a) E1a tester (KU-1978B)

Chromosome pairing in the $\mathrm{F}_{1}$ 's of the E1a tester was generally stable, with a small portion of PMC's showing precocious dissociation of the telocentric chromosomes. The fact that all the $\mathrm{F}_{1}$ 's formed no quadrivalent in at least $93 \%$ of the PMC's and no univalent indicated the similarity of this tester to LD 222 in chromosome structure. However, the critical pairing $\left[(t+t) 3^{\mathrm{V}}+12^{\prime \prime}\right]$ was observed in the $\mathrm{F}_{1}$ 's containing $2 \mathrm{~B}$ telosomes and $4 \mathrm{~B}$ telosomes by $6.7 \%$ and $4.2 \%$ of PMC's, respectively. Therefore, it is concluded that the E1a translocation type is almost the same, but a little differentiated from LD 222 by a reciprocal 
Table 1. Frequencies $(\%)$ of PMC's with multivalents in the $F_{1}$ 's between Kawahara's transiocation testers of $T$. dicoccoides and doubleditelosomics or ditelomonotelosomics for the A and B genome chromosomes of Emmer wheat

\begin{tabular}{|c|c|c|c|c|c|c|c|c|}
\hline \multirow{2}{*}{$\begin{array}{c}\mathrm{F}_{1} \text { telo- } \\
\text { somic for }\end{array}$} & \multirow{2}{*}{$\begin{array}{c}\text { No. of } \\
\text { PMC's } \\
\text { observed }\end{array}$} & \multicolumn{7}{|c|}{ Multivalents } \\
\hline & & $\begin{array}{c}(\mathrm{t}+\mathrm{t}) 1^{3} \\
{\left[\mathrm{t} 1^{2}\right.}\end{array}$ & $\begin{array}{c}(\mathrm{t}+\mathrm{t}) 1^{3}+1^{4} \\
\mathrm{t} 1^{2}+1^{4}\end{array}$ & $\begin{array}{c}(\mathrm{t}+\mathrm{t}) 1^{3}+2^{4} \\
\mathrm{t} 1^{2}+2^{4}\end{array}$ & $\begin{array}{c}(\mathrm{t}+\mathrm{t}) 3^{5} \\
\mathrm{t} 3^{4}\end{array}$ & $\begin{array}{c}(\mathrm{t}+\mathrm{t}) 3^{5}+1^{4} \\
\mathrm{t} 2^{4}+1^{4}\end{array}$ & $\begin{array}{c}(\mathrm{t}+\mathrm{t}) 5^{7} \\
\mathrm{t} 5^{6}\end{array}$ & $\begin{array}{c}1^{6}+(\mathrm{t}+\mathrm{t}) 1^{3} \\
\left.1^{6}+1^{2}\right]^{*}\end{array}$ \\
\hline \multicolumn{9}{|c|}{ (a) E1a tester (KU-1978) (2AL, 3BL, 5BL, 6BS)** } \\
\hline 2BLS & 163 & 93.3 & 0 & 0 & 6.7 & 0 & 0 & 0 \\
\hline $4 \mathrm{~B} \alpha \beta$ & 71 & 95.8 & 0 & 0 & 4.2 & 0 & 0 & 0 \\
\hline others*** & 143 & 95.4 & 4.6 & 0 & 0 & 0 & 0 & 0 \\
\hline \multicolumn{9}{|c|}{ (b) E1b tester (KU-108-3) (2AL, 3BL, 5BL, 6BS)** } \\
\hline $2 \mathrm{AL}$ & 134 & 71.6 & 0 & 0 & 28.4 & 0 & 0 & 0 \\
\hline 2BLS & 136 & 54.4 & 0.7 & 0 & 42.6 & 0 & 2.2 & 0 \\
\hline $3 \mathrm{BL}$ & 150 & 48.0 & 50.0 & 0 & 2.0 & 0 & 0 & 0 \\
\hline others & 158 & 60.0 & 39.4 & 0 & 0 & 0 & 0 & 0 \\
\hline \multicolumn{9}{|c|}{ (c) $\mathrm{E} 2$ tester (KU-109) $(2 \mathrm{AL}, 5 \mathrm{AL}, 6 \mathrm{AL}, 1 \mathrm{BS}, 3 \mathrm{BL}, 4 \mathrm{~B} \alpha, 5 \mathrm{BL})^{* *}$} \\
\hline 2BLS & 108 & 0 & 0 & 0 & 100.0 & 0 & 0 & 0 \\
\hline $3 \mathrm{BL}$ & 108 & 0 & 0 & 0 & 100.0 & 0 & 0 & 0 \\
\hline others & 105 & 1.9 & 97.7 & 0 & 0 & 0 & 0 & 0.3 \\
\hline \multicolumn{9}{|c|}{ (d) E3 tester $(\mathrm{KU}-195)(2 \mathrm{AL}, 5 \mathrm{AL}, 3 \mathrm{BL}, 5 \mathrm{BL})^{* *}$} \\
\hline $5 \mathrm{BL}$ & 119 & 0 & 0 & 0 & 100.0 & 0 & 0 & 0 \\
\hline 7BLS & 100 & 0 & 0 & 0 & 100.0 & 0 & 0 & 0 \\
\hline others & 105 & 6.4 & 91.6 & 2.0 & 0 & 0 & 0 & 0 \\
\hline \multicolumn{9}{|c|}{ (e) $\mathrm{E} 4$ tester $(\mathrm{KU}-8915 \mathrm{~A})(2 \mathrm{AL}, 3 \mathrm{AS}, 1 \mathrm{BS}, 5 \mathrm{BL}, 6 \mathrm{BS})^{* *}$} \\
\hline $2 \mathrm{BLS}$ & 107 & 0 & 77.6 & 0.6 & 1.3 & 20.0 & 0 & 0 \\
\hline 3BLS & 107 & 1.9 & 0 & 0 & 96.3 & 1.9 & 0 & 0 \\
\hline $4 \mathrm{~B} \alpha \beta$ & 146 & 2.1 & 0 & 0 & 95.9 & 2.1 & 0 & 0 \\
\hline others & 142 & 1.9 & 86.9 & 11.1 & 0 & 0 & 0 & 0 \\
\hline \multicolumn{9}{|c|}{ (f) E5 tester (KU-1945) (2AL, 3AL, 5AL, 3BL, 5BL, 6BS)** } \\
\hline 7ALS & 165 & 0 & 97.2 & 1.8 & 0 & 0 & 1.2 & 0 \\
\hline 2BLS & 146 & 1.4 & 96.6 & 0.7 & 0 & 0 & 1.4 & 0 \\
\hline $6 \mathrm{BS}$ & 183 & 4.4 & 0 & 0 & 95.6 & 0 & 0 & 0 \\
\hline 7BLS & 138 & 1.4 & 0 & 0 & 97.1 & 0 & 1.4 & 0 \\
\hline others & 170 & 1.6 & 96.8 & 1.5 & 0 & 0 & 0 & 0.1 \\
\hline \multicolumn{9}{|c|}{ (g) $\quad$ E6 tester (KU-1952) (2AL, 6AS, 3BL, 5BL)** } \\
\hline 1ALS & 120 & 0 & 0 & 0 & 100.0 & 0 & 0 & 0 \\
\hline 5ALS & 107 & 0 & 0 & 0 & 93.5 & 6.5 & 0 & 0 \\
\hline others & 109 & 5.6 & 90.8 & 3.6 & 0 & 0 & 0 & 0 \\
\hline
\end{tabular}

* Multivalents in monotelodisomic $\mathrm{F}_{1}$ 's were in brackets.

** $\mathrm{F}_{1}$ 's were monotelodisomic for the chromosome arms in parentheses.

*** Pooled data of all the non-critical $\mathrm{F}_{1}$ 's. 
translocation of small segments between $2 \mathrm{~B}$ and 4B. Nishikawa (1967) showed that LD 222 has almost the same A and B genome as those of Chinese Spring. Kawahara (1984) pointed out that there are minor chromosomal variations among the strains of type E1. Kawahara (1991) also reported that the $F_{1}$ of the E1a tester and Chinese Spring rarely formed a quadrivalent.

(b) E1b tester (KU-108-3)

A quadrivalent, mostly in a chain of four configuration, was formed in 22.4$65.9 \%$ (40\% on the average) of the PMC's in the non-critical $\mathrm{F}_{1}$ lines. The critical pairing was observed in three $\mathrm{F}_{1}$ 's, respectively containing $2 \mathrm{~A}$ telosome, $2 \mathrm{~B}$ telosomes and $3 \mathrm{~B}$ telosome. The $\mathrm{F}_{1}$ with the $2 \mathrm{~B}$ telosomes formed $(t+t) 3^{\mathrm{V}}$ the most frequently and also formed a septivalent $(t+t) 5^{\mathrm{VII}}$ in $2.2 \%$ of the PMC's. Then, it seems reasonable to consider that $2 \mathrm{~A}, 2 \mathrm{~B}$ and $3 \mathrm{~B}$ are comprised in the sexivalent, and that $2 \mathrm{~B}$ and $2 \mathrm{~A}$ are included in most of the quadrivalent. Therefore, the E1b tester is concluded to be different from durum LD222 by two translocations, the small one between $2 \mathrm{~B}$ and $3 \mathrm{~B}$ and the large one between $2 \mathrm{~A}$ and $2 \mathrm{~B}$.

(c) E2 tester (KU-109)

A quadrivalent was formed in almost all (97.7\%) PMC's in the non-critical $\mathrm{F}_{1}$ 's of the E2 tester. The quadrivalent occurred in a ring of four configuration in $63 \%$ of the PMC's. The $\mathrm{F}_{1}$ 's containing $2 \mathrm{~B}$ telosomes and $3 \mathrm{~B}$ telosome showed the critical pairing in all the PMC's. Evidently long segments must have been interchanged between $2 \mathrm{~B}$ and $3 \mathrm{~B}$ in the $\mathrm{E} 2$ tester. Very rare occurrence of $1^{\mathrm{VI}}+$ $11^{\prime \prime}$ indicated that there was another small translocation involving $2 \mathrm{~B}$ or $3 \mathrm{~B}$. Considering that there were one major and one minor translocations with one chromosome in common between E1b and E2 (Kawahara 1984), 2B seems more likely involved than $3 \mathrm{~B}$.

(d) E3 tester (KU-195)

One major quadrivalent occurred in $93.6 \%$, and two quadrivalents in $2.0 \%$, of the PMC's in the non-critical $F_{1}$ 's, nearly half of the major quadrivalent being ring. The major quadrivalent rarely dissociated into $1^{\prime \prime \prime}+1^{\prime}$. The critical pairing occurred in the $\mathrm{F}_{1}$ 's respectively containing $5 \mathrm{~B}$ telosome and $7 \mathrm{~B}$ telosomes. This indicated that there was a major translocation between $5 \mathrm{~B}$ and $7 \mathrm{~B}$ in the $\mathrm{E} 3$ tester. No information was obtained on the minor translocation. Kawahara (1984) also observed two quadrivalents in $6.1 \%$ of the PMC's in the $F_{1}$ between the E1 tester and the E3 tester.

(e) E4 tester (KU-8915A)

The $\mathrm{F}_{1}$ between this tester and $5 \mathrm{~A}$ telosomic line was not available. Almost all the PMC's in the non-critical $F_{1}$ 's had at least one quadrivalent, and about $11 \%$ of them had another quadrivalent. Over $60 \%$ of quadrivalents were ring. In the PMC's with only one quadrivalent the ring-of-four configuration was predominant over the chain-of-four configuration. The critical pairing occurred in $21.3 \%$, $98.2 \%$ and $98.0 \%$ of the PMC's of three $\mathrm{F}_{1}$ 's containing the telosomes of $2 \mathrm{~B}, 3 \mathrm{~B}$ 
and $4 \mathrm{~B}$, respectively. In the three $\mathrm{F}_{1}$ 's the second quadrivalent was observed in $20 \%, 1.9 \%$ and $2.1 \%$ of the PMC's, respectively. It was evident that chromosomes $3 \mathrm{~B}$ and $4 \mathrm{~B}$ were involved in the major translocation and $2 \mathrm{~B}$ was involved in the smaller translocation.

(f) E5 tester (KU-1945)

A quadrivalent was formed in nearly all the PMC's in the non-critical $F_{1}$ 's, but two quadrivalents were found rarely. About half of the quadrivalents were in a ring configuration. A chain of six chromosomes was, although very rarely, observed in non-critical line of $4 \mathrm{~A}$ telosomes. The critical pairing was formed in almost all the PMC's of $\mathrm{F}_{1}$ 's containing $6 \mathrm{~B}$ telosome and $7 \mathrm{~B}$ telosomes, respectively, and $(\mathrm{t}+\mathrm{t}) 5^{\mathrm{VII}}$ pairing occurred rarely in the $\mathrm{F}_{1}$ 's respectively containing the telosomes of $7 \mathrm{~A}, 2 \mathrm{~B}$ and $7 \mathrm{~B}$. With regard to chromosome pairing, lines $7 \mathrm{~A}$ and $2 \mathrm{~B}$ were similar to the non-critical lines, with exception of rare occurrence of $(t+$ t) $5^{\mathrm{VII}}$. These facts suggested that the fairy long segments were interchanged between chromosomes $6 \mathrm{~B}$ and $7 \mathrm{~B}$, and that a small translocations occurred among $7 \mathrm{~A}, 2 \mathrm{~B}$ and $7 \mathrm{~B}$.

(g) E6 tester (KU-1952)

About $95 \%$ of PMC's in the non-critical $\mathrm{F}_{1}$ 's had at least one quadrivalent, over $60 \%$ of which was in a ring of four. The critical pairing was formed in all the PMC's in the $\mathrm{F}_{1}$ 's of the $1 \mathrm{~A}$ and $5 \mathrm{~A}$ telocentric lines. This indicated that the fairy long segments were reciprocally translocated between chromosomes $1 \mathrm{~A}$ and $5 \mathrm{~A}$. The results described above is summarized in Table 2 .

Table 2. Chromosomes involved in Kawahara's six types of translocation in Triticum dicoccoides in relation to $\mathrm{LD} 222$

\begin{tabular}{ccc}
\hline \hline Translocation type & Major & Minor \\
\hline E1 & --- & $2 \mathrm{~B}-4 \mathrm{~B}$ \\
E1b & 2A- 2B & 2B- 3B \\
E2 & 2B- 3B & $2 \mathrm{~B}-?$ \\
E3 & $5 \mathrm{~B}-7 \mathrm{~B}$ & $?-?$ \\
E4 & $3 \mathrm{~B}-4 \mathrm{~B}$ & $2 \mathrm{~B}-?$ \\
E5 & $6 \mathrm{~B}-7 \mathrm{~B}$ & $7 \mathrm{~A}-2 \mathrm{~B}-7 \mathrm{~B}$ \\
E6 & $1 \mathrm{~A}-5 \mathrm{~A}$ & $?-?$ \\
\hline
\end{tabular}

Kawahara (1984) inferred genomes to which the chromosomes involved in his six types of translocation belonged. Types E2, E3 and E5 had translocations between the B genome chromosomes, type E4 between the A and B genome chromosomes, and type E6 between the A genome chromosomes. He also showed that E1b was different from E1a by a minor translocation between the A and $\mathrm{B}$ genome chromosome. The present result completely agreed with Kawa- 
hara's inferrence except for type E4. Our result evidently showed the presence of a translocation between chromosomes $3 \mathrm{~B}$ and $4 \mathrm{~B}$ in type E4. There is no likely explanation for this discrepancy. From the result in Table 2 a sexivalent would be expected to occur in the $\mathrm{F}_{1}$ 's between E2 and E4 and between E3 and E5. This was observed by Kawahara (1984).

The chromosome structure of LD 222 is prevailing in cultivated form of Emmer group (Nishikawa et al., 1986). This implies that E1a, the most common translocation type among wild Emmer, is also the major type among cultivated Emmer, though the two forms are differentiated by a minor translocation. Although Nishikawa et al. (1986) identified three translocation types other than LD 222 type in cultivated form of Emmer group, they were not found in wild Emmer.

\section{REFERENCES}

Chapman, V. and Riley, R. (1966). The allocation of the chromosomes of Triticum aestivum to the A and B genomes and evidence on genome structure. Can. J. Genet. Cytol. 8, 57-63.

Kawahara, T. (1984). Studies of interspecific structural differentiation of chromosomes in the wild tetraploid wheats. Doctor Thesis, Kyoto Univ. Kyoto.

Kawahara, T. (1986). Identification of reciprocal translocation chromosome types in the Emmer wheats. II. Thirty eight strains of Triticum dicoccoides Körn. with fundamental chromosome structure. Wheat Inform. Serv. 61, 1-6.

Kawahara, T. (1987). Identification of reciprocal translocation chromosome types in Emmer wheats. III. Six chromosome types in Triticum dicoccoides. Jpn. J. Genet. 62, 197-204.

Kawahara, T. and Tanaka, M. (1983). Chromosomal interchanges and the evolution of the B and G genomes. Proc. 6th Int. Wheat Genet. Symp. Kyoto, pp. 977-981.

Nishikawa, K. (1967). Introduction of telocentric chromosomes of Chinese Spring into Triticum durum. Wheat Inform. Serv. 25, 5-6.

Nishikawa, K., Takagi, A., Ban, T., Otuka, H. and Furuta, Y. (1986). Spontaneous reciprocal translocations in cultivated form of Emmer wheat. Jpn. J. Genet. 61, 361-370. 